\title{
Ultra-rapid earth rotation determination with VLBI during CONT11 and CONT14
}

\author{
Rüdiger Haas $^{1}{ }_{\mathbb{D}} \cdot$ Thomas Hobiger $^{1} \cdot$ Shinobu Kurihara $^{2} \cdot$ Tetsuya Hara $^{2}$
}

Received: 29 April 2016 / Accepted: 9 November 2016 / Published online: 17 November 2016

(C) The Author(s) 2016. This article is published with open access at Springerlink.com

\begin{abstract}
We present earth rotation results from the ultrarapid operations during the continuous VLBI campaigns CONT11 and CONT14. The baseline Onsala-Tsukuba, i.e., using two out of the 13 and 17 stations contributing to CONT11 and CONT14, respectively, was used to derive UT1-UTC in ultra-rapid mode during the ongoing campaigns. The latency between a new observation and a new UT1-UTC result was less than 10 min for more than $95 \%$ of the observations. The accuracy of the derived ultra-rapid UT1-UTC results is approximately a factor of three worse than results from optimized one-baseline sessions and/or complete analysis of large VLBI networks. This is, however, due to that the one-baseline picked from the CONT campaigns is not optimized for earth rotation determination. Our results prove that the 24/7 operation mode planned for VGOS, the next-generation VLBI system, is possible already today. However, further improvements in data connectivity of stations and correlators as well in the automated analysis are necessary to realize the ambitious VGOS plans.
\end{abstract}

Keywords VLBI · Earth rotation parameter · UT1-UTC . Ultra-rapid operations · VGOS · 24/7 mode

Rüdiger Haas

rudiger.haas@chalmers.se

Thomas Hobiger

thomas.hobiger@chalmers.se

Shinobu Kurihara

kurihara-s96bb@mlit.go.jp

Tetsuya Hara

hara-t96xs@mlit.go.jp

1 Onsala Space Observatory, Department of Earth and Space Sciences, Chalmers University of Technology, 43992 Onsala, Sweden

2 Geospatial Information Authority of Japan, Tsukuba, Japan

\section{Introduction}

Geodetic Very Long Baseline Interferometry (VLBI) is unique among the space geodetic techniques since it directly relates the Celestial Reference Frame (CRF) and the Terrestrial Reference Frame (TRF). It is thus very sensitive to the earth rotation parameters that describe the transformation between the two frames. In particular, it is the only space geodetic technique that can determine the earth rotation angle, which is usually expressed as UT1-UTC, i.e., the difference between astronomical time and atomic time. Since this parameter is important in many aspects for science and technology, there is a continuous interest in accurate and timely UT1-UTC values. The exchange of angular momentum between the atmosphere, geophysical fluids, and the solid earth causes variations in UT1-UTC, see Barnes et al. (1983). Therefore, accurate UT1-UTC results allow detailed studies of geodynamical phenomena and excitation, see Brzezinski (2012). Also for the calculation of precise satellite orbits, UT1-UTC is a very crucial parameter, see Bradley et al. (2015). Global Navigation Satellite Systems (GNSS) need VLBI-determined UT1-UTC since satellite techniques can only determine the time derivative of UT1UTC.

Since several years, the International VLBI Service for Geodesy and Astrometry (IVS) organizes since so-called Intensive (INT) sessions with the aim to determine UT1UTC on a regular basis. These observation sessions usually last $1 \mathrm{~h}$ and involve one baseline only. Currently, there are three INT series observed on different days of the week and with different participating stations, see Fig. 1. Generally, the basic configuration is one long east-west-oriented baseline. For the INT-1 series, which is observed on weekday evenings UT time, usually the stations Wettzell in Germany and Kokee on Hawaii are involved. For INT-2, which is 

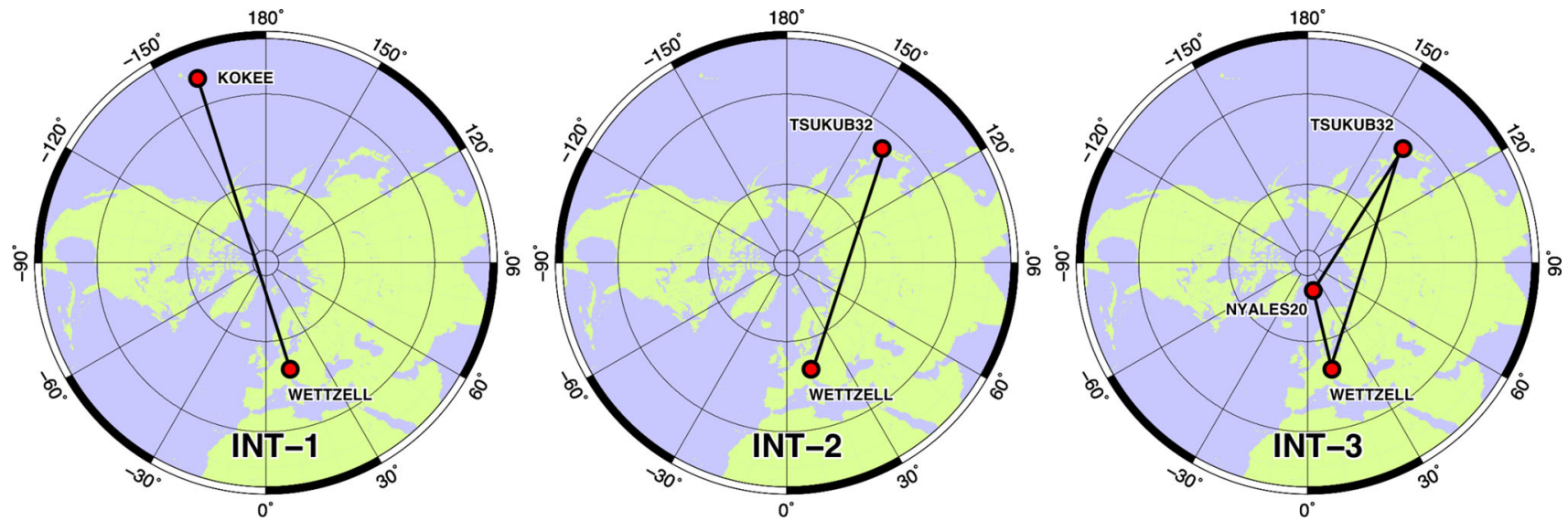

Fig. 1 Baselines used for the IVS Intensive series: INT-1 (left) are observed on weekdays (left plot), INT-2 (middle) on weekends, and INT-3 (right) on Monday mornings

observed on weekends, usually the stations Wettzell in Germany and Tsukuba in Japan are involved. Occasionally, these standard configurations are modified due to station maintenance issues, and/or augmented by additional stations. The INT-3 series is observed on Monday mornings UT time and involves usually Wettzell, Kokee and Ny-Ålesund, Spitsbergen. Depending on the data shipment times and correlation times, final UT1-UTC values are today usually determined within several hours after the actual observations are finished.

The next-generation VLBI system, the VLBI Global Observing System (VGOS), aims at so-called 24/7 operations to determine earth rotation continually and with very low latency (Petrachenko et al. 2009). Nothnagel et al. (2016) describe the IVS strategic plan for VGOS and the planned future IVS earth rotation parameter products in terms of their latency and accuracy. For UT1-UTC products with a latency as low as $30 \mathrm{~min}$, an accuracy of $7 \mu \mathrm{s}$ is expected. Accuracies of 5 and $3 \mu$ s are anticipated for corresponding products with a latency of 3 and $12 \mathrm{~h}$, respectively. For so-called final products, an accuracy of $1 \mu \mathrm{s}$ is expected.

To achieve UT1-UTC with low latency, beginning in 2007 the Geospatial Information Authority of Japan (GSI) and the Onsala Space Observatory (OSO) in Sweden started a collaboration project aiming at determining UT1-UTC in near real time (Sekido et al. 2008). In the beginning of this project, dedicated 1-h-long one-baseline experiments were observed periodically using the VLBI stations Onsala (Sweden) and Tsukuba (Japan). The observed VLBI data were sent in real time via the international optical fibre backbone to the correlator at Tsukuba where the data were correlated with a software correlator and analyzed in near real time with the c5++ VLBI data analysis software. This allowed the production of UT1-UTC results with very low latency. These sessions used data rates of 128 or $256 \mathrm{Mb} / \mathrm{s}$. The latency between the 1-h-long observation session at the stations and the determination of UT1-UTC is on the order of a few minutes (Matsuzaka et al. 2008). This meant that ultra-rapid determination of UT1-UTC became possible. It was shown that the agreement of these ultra-rapid UT1-UTC sessions with respect to the IERS C04 series (Bizouard and Gambis 2011) are on the same level as the standard INT sessions (Haas et al. 2010; Koyama et al. 2010).

An offline version of this strategy was adopted in 2009 for the regular VLBI intensive series INT-2. Since March 2010, the INT-2 uses real-time e-transfer too, and since June 2010 also automated analysis. It was shown by Luzum and Nothnagel (2010) that this leads to improved UT1 predictions.

Starting in 2009 the ultra-rapid approach was applied to regular 24-h-long IVS VLBI sessions that involve Tsukuba and Onsala. These sessions are not optimized for the determination of UT1-UTC, but for general earth rotation parameter determination in global VLBI networks. It was shown that it was possible to determine ultra-rapid UT1-UTC results during ongoing 24-h sessions (Matsusaka et al. 2010). As a consequence of this, the strategy was applied to the two continuous VLBI campaigns CONT11 and CONT14. These efforts were meant as a pilot project for the next-generation VLBI system, the VLBI Global Observing System (VGOS).

Section 2 explains the strategy developed to achieve ultrarapid earth rotation parameters from geodetic VLBI. The application of this strategy to CONT11 and CONT14 is described in Sect. 3. The corresponding results are presented and discussed in Sect. 4, and Sect. 5 concludes the paper.

\section{The strategy for ultra-rapid earth rotation determination}

The strategy to determine ultra-rapid earth rotation parameters from geodetic VLBI observations is based on using (a) real-time data transfer to the correlator, (b) (near) realtime data correlation, and (c) (near) real-time data analysis. Ground-breaking work in this direction was already per- 
formed in the late 1990 in Japan (e.g., Koyama et al. 1998), where domestic Japanese VLBI operations could be automated. However, only the connection of VLBI stations worldwide to the international optical fibre backbone made it possible to perform meaningful ultra-rapid earth rotation measurements. While the astronomical VLBI community uses real-time correlation on a regular basis, such data processing is still very rare in the geodetic VLBI community since the correlators operating for geodetic VLBI are in general not as well equipped in terms of optical fibre connections and data rates, as the correlators used for astronomical VLBI are. For the ultra-rapid VLBI sessions on the OnsalaTsukuba baseline described in this paper, the data correlation was performed at the Tsukuba VLBI correlator. The observational data from Onsala were thus real time transferred to the Tsukuba correlator using the tsunami UDP protocol (Tsunami 2009). Similarly, the observational data from the Tsukuba station were transferred in real time to the Tsukuba correlator. At the Tsukuba correlator, the data of both stations were stored first on a computer cluster. Since the raw VLBI data were in two different data formats, the Onsala data had to be converted first before being fed to the Tsukuba correlator. This conversion was done in near real time. After that the Onsala-Tsukuba data were correlated and VLBI delays for each scan were determined. These delays were then fed into the c5++ VLBI data analysis software (Hobiger et al. 2010) to estimate UT1-UTC.

For the analysis, several different strategies were tested during the years. For dedicated 1-h-long intensive-type sessions that were optimized for earth rotation estimation, all available delays produced from the 1-h observations were used together for the data analysis. This means that these sessions were scheduled in a similar way as the standard IVS INT sessions, i.e., optimized to estimate the earth rotation angle with $1 \mathrm{~h}$ of observations on a single VLBI baseline, and with data rates of $128 \mathrm{and} /$ or $256 \mathrm{Mb} / \mathrm{s}$. This scheduling approach leads to about 30 observations per hour. However, for the ultra-rapid UT1-UTC determinations during standard 24-h IVS-sessions, a different approach needed to be developed, since the schedules of these sessions were optimized for network observations and not for just one baseline. This leads to only 4-7 observations per hour on the baseline Onsala-Tsukuba. Thus, the geometry resulting from $1 \mathrm{~h}$ of observations on only the one baseline Onsala-Tsukuba was not suitable for UT1-UTC determination. To solve this difficulty, sliding-window approaches were applied using either a fixed number of observations or a fixed time interval with delay observations for the analysis. For example, a fixed window of 35 scans was used, or a fixed time window of, e.g., 3 or $6 \mathrm{~h}$ length was used for the analysis. The length of the time window was chosen to assure a sufficiently large number of observations, about 30, and a suitable geometry. These windows were slid through the session, i.e., as soon a new delay observation was produced by the correlator, it was added to the sliding window and the "oldest" delay observation was removed from the window, with keeping the size of the window the same, either in terms of number of scans or interval length. This approach made it possible to continually determine UT1-UTC during an ongoing VLBI session in near real time. The latency between a new observation and a new UT1-UTC result depends on the length of a scan, and thus the time to convert the data, correlate and then to perform the analysis. Usually, latencies below 15 min can be achieved.

\section{Ultra-rapid operations during CONT11 and CONT14}

The continuous VLBI campaigns CONT11 and CONT14 were organized by the IVS to acquire state-of-the-art VLBI data over a time period of 15 days each. The campaigns aimed at demonstrating the highest accuracy of which the VLBI system was capable, with a special focus on earth rotation. CONT11 was observed on September 15 through 29, 2011 and CONT14 May 6 through 20, 2014. Both campaigns covered 15 continuous 24-h sessions starting at 00:00 UT. The number of stations participating were 13 and 17 for CONT11 and CONT14, respectively. The corresponding network distributions are shown in Fig. 2. Since Onsala and Tsukuba were involved in both CONT campaigns, these could be used to determine ultra-rapid earth rotation using the approach described in Sect. 2. The continuity aspect of the CONTs makes them highly relevant as pilot project for the upcoming VGOS system, since the 24/7 operations could be tested effectively. For CONT11 and CONT14, the average number of observations per hour on the Onsala-Tsukuba baseline were 6.7 and 8.5 observations/h, respectively.

The operations of CONT11 and CONT14 worked generally very well at both stations. The observational data were streamed in real time with $512 \mathrm{Mb} / \mathrm{s}$ from Onsala and Tsukuba to the Tsukuba correlator. During CONT11, there was, however, one day of outage (21 September) at Tsukuba due to the Typhoon Roke during which the telescope had to stow. Thus, during this day, no ultra-rapid UT1-UTC could be determined. Onsala on the other hand suffered from small power outages on September 25 and 26. During CONT14, no major disturbances in the ultra-rapid operations occurred.

For $50 \%$ of the scans, the latency between the observation and a new UT1-UTC result was less than 5 min. For $95 \%$ of the scans it was less than $10 \mathrm{~min}$, and for all scans it was less than $15 \mathrm{~min}$.

\section{Results from CONT11 and CONT14}

Figure 3 depicts the UT1-UTC results for the two CONT campaigns. Shown are the ultra-rapid results (CONT-UR) 


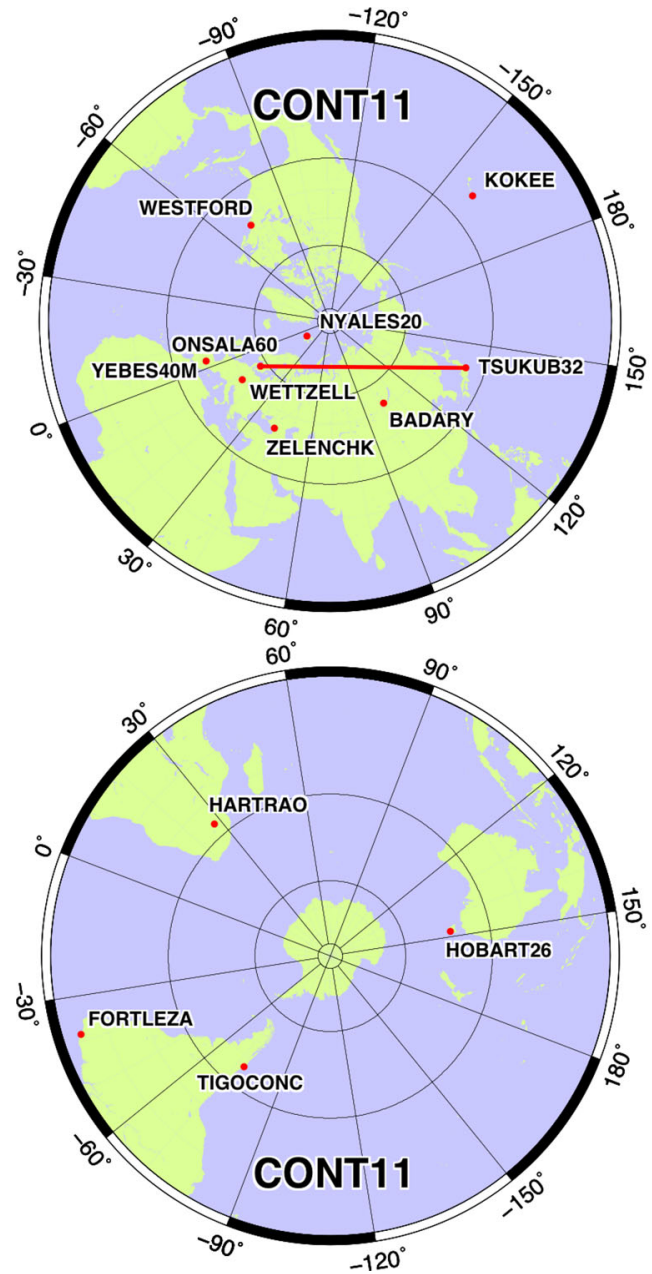

Fig. 2 Network distribution for CONT11 (left column) and CONT14 (right column). Shown are the northern (top row) and southern hemisphere (bottom row). The northern hemisphere station distribution in the two CONT campaigns is almost identical while the south-

together with the results derived from the INT sessions observed during the two CONTs, post-processing results with 1-h temporal resolution from the analysis of the complete CONT networks (CONT-1h), as well as the IVS quarterly solution with 24-h temporal resolution (IVS-Q). Results of three different INT analyses are presented, by the IVS analysis groups at the Bundesamt für Kartographie und Geodäsie (BKG), the Goddard Space Flight Center (GSFC), and the United States Naval Observatory (USNO). The ultra-rapid results presented were determined with a sliding-window approach using a window size of $6 \mathrm{~h}$. The ultra-rapid results are displayed at the correct level, while all other data sets are offset by $-5 \mathrm{~ms}$ with respect to the corresponding previous data set, to improve visibility of the graphs.

For CONT11, there were only INT-1 sessions during the CONT, i.e., on the baseline Wettzell-Kokee. On the contrary,

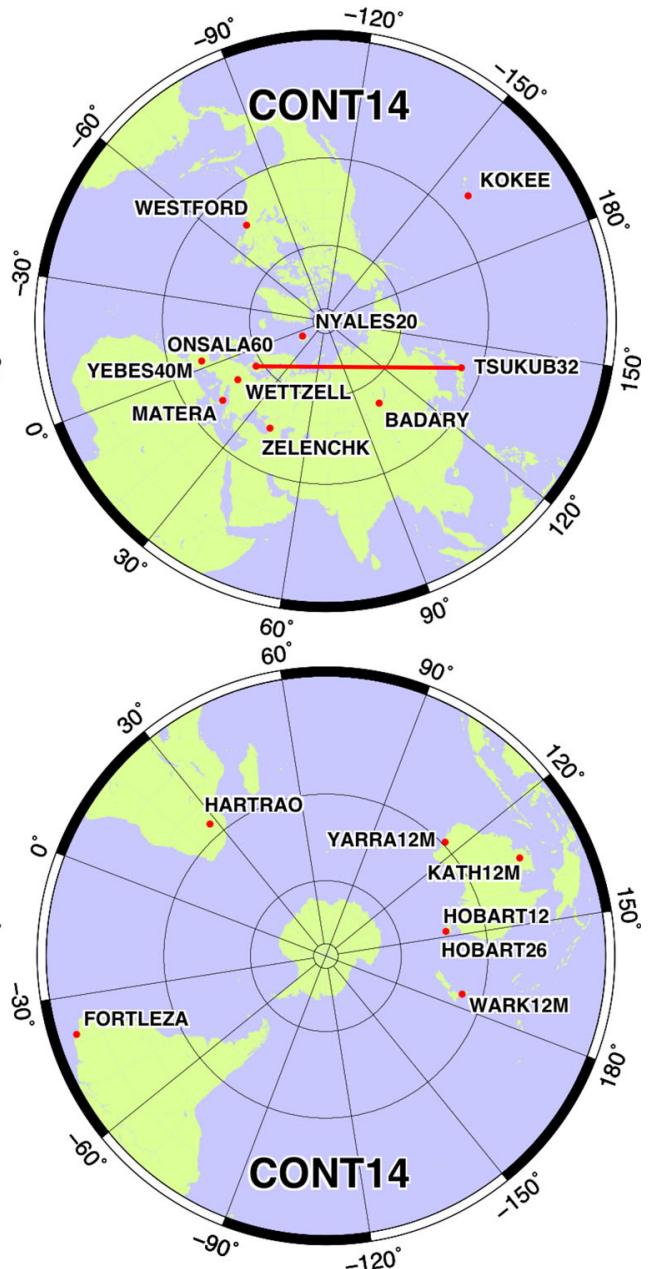

ern hemisphere station distribution is quite different. The baseline ONSALA60-TSUKUB32 that was used for ultra-rapid earth rotation determination during both CONTs is depicted in red

during CONT14 there were both INT-1 (baseline WettzellKokee) during the weekdays, and INT-2 (baseline WettzellTsukuba) during the weekends May 10/11 and May 17/18. Thus, for CONT14 there are no delay observations on the Onsala-Tsukuba baseline during these four INT-2 sessions. However, since the sliding window used was larger than the 1h outage, there are nevertheless ultra-rapid UT1-UTC values reported during these gaps.

There is a large gap in the ultra-rapid results for CONT11 on September 21, because the Tsukuba telescope was stowed because of the Typhoon. Also the smaller gaps on September 25 and 26 that were caused by power outages at Onsala are visible.

In general, it can be noted that the different data sets show a very similar behavior and that the curves follow each other nicely. Referring the data sets to a common reference gives more insight into the details. As common reference, the 

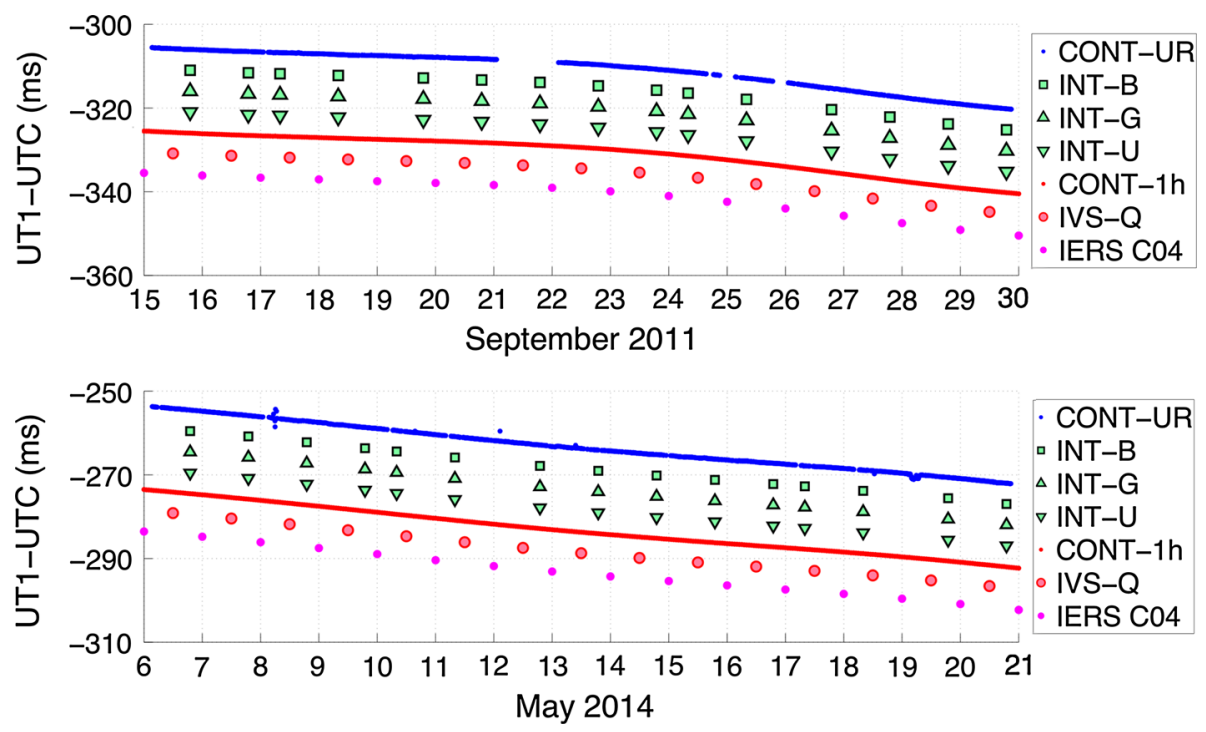

Fig. 3 Results of UT1-UTC for CONT11 (top) and CONT14 (bottom). Shown from top to bottom are the CONT ultra-rapid results (CONT$\mathrm{UR}$, blue dots), the INT results from the analysis of BKG (INT-B, green squares), GSFC (INT-G, green upward triangles), and USNO (INT-U, green downward triangles), as well as the CONT post-processing results with 1-h temporal resolution (CONT-1h, small red dots), the IVS quarterly solutions with 24-h temporal resolution (IVS-Q, large red dots) and the IERS $08 \mathrm{C} 04$ results (small magenta dots). The data sets are offset by multiples of $5 \mathrm{~ms}$ from each other to improve visibility
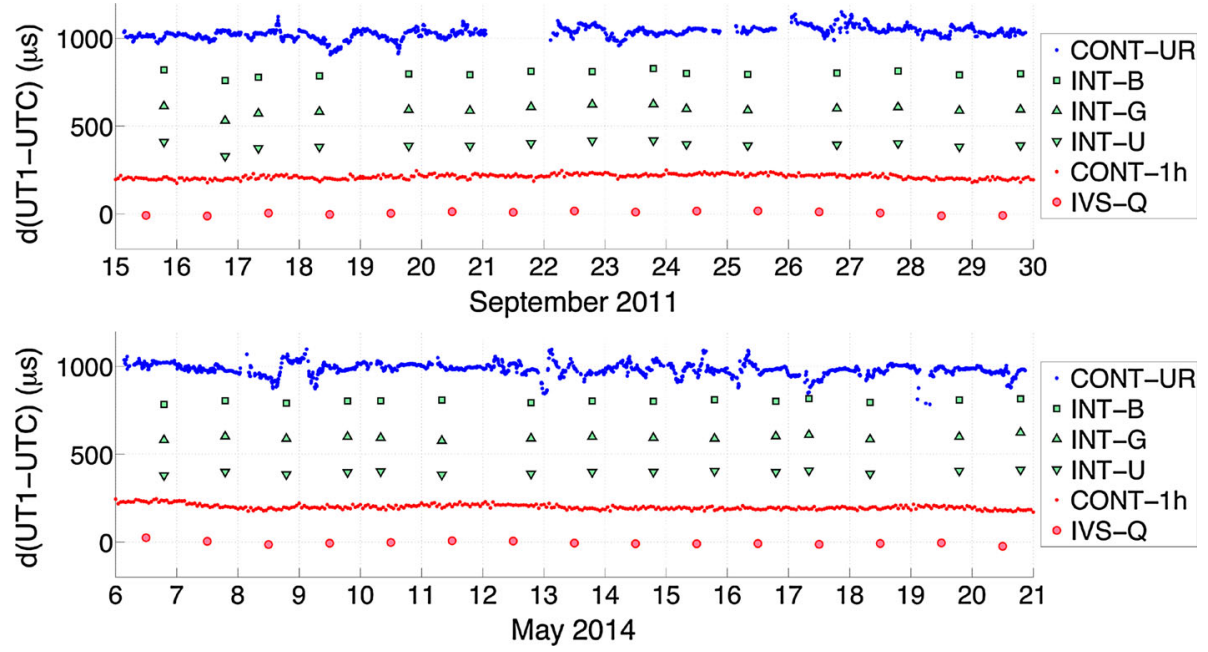

Fig. 4 Results of UT1-UTC residuals with respect to the IERS 08 C04 series for CONT11 (top) and CONT14 (bottom). Shown from top to bottom are the CONT ultra-rapid results (CONT-UR, blue dots), the INT results from the analysis of BKG (INT-B, green squares), GSFC (INT-G, green upward triangles), and USNO (INT-U green downward

IERS 08 C04 data were used and the corresponding residuals are presented in Fig. 4.

The UT1-UTC residuals presented in Fig. 4 clearly show that the ultra-rapid results derived from one baseline only have a larger variability than the results derived from analysis of the complete network data, both the post-processed results with 1-h temporal resolution and the IVS quarterly solution with 24-h temporal resolution. Also the INT results show a considerable scatter, and have of course a much worse tem- triangles), as well as the CONT post-processing results with 1-h temporal resolution (CONT-1h, small red dots) and the IVS quarterly solutions with 24-h temporal resolution (IVS-Q, large red dots). The data sets are offset by multiples of $200 \mu$ s from each other to improve visibility

poral resolution as both the ultra-rapid results as well the 1-h post-processed network results. There are some small deviations of the ultra-rapid results probably caused by failure of the automated ambiguity resolution that is part of the automated processing. For the ultra-rapid CONT11 and CONT14 analysis, robust methods (Kareinen et al. 2016) were not part of the automated analysis chain yet.

Table 1 summarizes statistical information of these comparisons in terms of root-mean-square differences (rms), 
Table 1 Information on the root-mean-square difference (rms), the bias (bias) and the standard deviation $(\sigma)$ of the UT1-UTC results with respect to the IERS C04 series

\begin{tabular}{|c|c|c|c|c|c|c|}
\hline \multirow[b]{2}{*}{ Series } & \multicolumn{3}{|c|}{ CONT11 } & \multicolumn{3}{|c|}{ CONT14 } \\
\hline & Rms & Bias & $\sigma$ & Rms & Bias & $\sigma$ \\
\hline CONT-UR (ultra-rapid) & 44.9 & +31.9 & 31.6 & 37.9 & -15.4 & 34.7 \\
\hline INT-B (BKG analysis) & 16.6 & -1.2 & 16.5 & 9.2 & +2.8 & 8.7 \\
\hline INT-G (GSFC analysis) & 22.9 & -6.0 & 22.1 & 12.1 & -4.4 & 11.3 \\
\hline INT-U (UNSO analysis) & 22.9 & -9.9 & 20.6 & 9.7 & -3.3 & 9.1 \\
\hline CONT-1h (1 h resolution) & 17.7 & +11.7 & 13.3 & 14.3 & 0.0 & 14.3 \\
\hline IVS-Q (IVS quarterly solution) & 10.5 & +3.3 & 10.0 & 11.7 & -4.1 & 10.9 \\
\hline
\end{tabular}

All values are given in $\mu \mathrm{s}$ biases, and standard deviations. The IVS quarterly results agree in terms of standard deviation with the IERS 08 C04 on the level of 10-11 $\mu$ s and show biases of less than $5 \mu \mathrm{s}$. The post-processed network results with 1-h temporal resolution agree slightly less good, in terms of standard deviation by a factor of 1.3-1.4 approximately. While there is no bias for CONT14, there is one of almost $12 \mu \mathrm{s}$ for CONT11. The INT results agree with IERS $08 \mathrm{C} 04$ in terms of standard deviation during CONT11 on the level of 16-22 $\mu$ s, but for CONT14 on the level of 9-12 $\mu \mathrm{s}$. The biases for CONT11 are between 1 and $10 \mu$ s while they are around 3-4 $\mu$ s for CONT14. The ultra-rapid results show both the largest biases and standard deviations on the order of $15-32$ and 32-35 $\mu$ s, respectively. We can conclude from these comparisons that UT1-UTC derived from the analysis of networks with many globally well-distributed stations agree better with IERS 08 C04 than results derived on a single baseline. We also see that results derived from single baseline observations that were optimized for a single baseline agree better with IERS 08 C04 than results from single baseline analysis of observations that were not optimized for this purpose.

While the ultra-rapid results are about a factor of three worse than the results from the INT sessions, they are still better than UT1-UTC predictions. According to the IERS Bulletin-A, the prediction accuracy degrades as a function of time in days (D) since the last official IERS UT1-UTC value by

$\sigma_{\mathrm{UT} 1-\mathrm{UTC}}=250 \cdot D^{0.75}[\mu \mathrm{s}]$.

This means that after 6,12 , and $18 \mathrm{~h}$, uncertainties on the order of 88,149 and $202 \mu \mathrm{s}$ are to be expected for UT1UTC predictions. The ultra-rapid results are better than this as confirmed by rms differences smaller than $50 \mu \mathrm{s}$ with respect to IERS $08 \mathrm{C} 04$.

\section{Conclusions and outlook}

The experience gained from the ultra-rapid operations during CONT11 and CONT14 shows that it is possible already today to operate VLBI in $24 / 7$ mode. This is the planned and anticipated VGOS operation mode and requires real-time data transfer to a correlator, near real-time correlation and near real-time data analysis. This strategy is possible today not only for both single baseline observations, but also for network operations (Kokado et al. 2012). However, the latter has so far only been demonstrated for a restricted number of participating stations and not yet for longer than $24 \mathrm{~h}$. Furthermore, so far all real-time operations have been done with rather moderate data rates of less than $1 \mathrm{~Gb} / \mathrm{s}$, which is much lower than the planned data rates for VGOS.

It is anticipated that the currently existing strategies for automated analysis (Hobiger et al. 2010; Kareinen et al. 2015) will be extended in the near future from single baseline observations to network observations. In this context, it is highly desirable to also include robust analysis strategies, as described by Kareinen et al. (2016).

The achieved accuracies from the CONT ultra-rapid single baseline operations are roughly a factor three worse than the results from both dedicated one-baseline sessions and/or the complete analysis of network sessions. This is due to that the CONT observations on the one baseline were not optimized for earth rotation determination. The number of observations per time unit and the geometrical distribution of the observations on this single baseline were not optimal, leading to worse results than for dedicated INT sessions. However, the ultra-rapid results are better than the uncertainty of UT1-UTC predictions communicated by IERS (cf. Eq. 1).

It is expected that future VGOS sessions will be network sessions that are optimized for earth rotation determination and that these will be operated and analyzed in ultra-rapid network mode, thus providing highly accurate earth rotation results. The new VGOS telescopes will allow to increase the number of observations per time by more than a factor of 5 , compared to, e.g., CONT14. Thus, it can be expected that even for single baselines in the VGOS network, already during $1 \mathrm{~h}$ a suitable number of observations and geometry can be achieved, to be able to determine earth rotation parameters with acceptable accuracy. Further improvement is expected when using more stations. 
One of the biggest challenges for VGOS will be the large number of stations [on the order of 16-24 (Petrachenko et al. 2014)] and the large amount of data to be sent to the correlators in real time. VGOS aims at data rates of $16 \mathrm{~Gb} / \mathrm{s}$ (Petrachenko et al. 2014), which is today and in the near future impossible to transfer in real time to the IVS correlators. Though some stations have already today optical fibre connections with capacities of $10 \mathrm{~Gb} / \mathrm{s}$ and more, this is not the case for the IVS correlators. The IVS correlators will need to improve their optical fibre connections drastically to be able to receive real-time data transfer at VGOS data rates from two or more stations.

The amount of data per station to be transferred to the correlators will be several tens of TB per day (Petrachenko et al. 2014). This means that the currently available connections to the optical fibre backbone for the stations, and in particular for the correlators, have to be improved also for the case of offline e-transfer.

Acknowledgements We thank the three reviewers for their valuable and constructive comments which helped to improve the paper. The International VLBI Service for Geodesy and Astrometry is acknowledged for providing the observational data (Behrend 2013).

Open Access This article is distributed under the terms of the Creative Commons Attribution 4.0 International License (http://creativecomm ons.org/licenses/by/4.0/), which permits unrestricted use, distribution, and reproduction in any medium, provided you give appropriate credit to the original author(s) and the source, provide a link to the Creative Commons license, and indicate if changes were made.

\section{References}

Barnes RTH, Hide R, White AA, Wilson CA (1983) Atmospheric angular momentum fluctuations, length-of-day changes and polar motion. Proc R Soc Lond Ser A 387(1792):31-73

Behrend D (2013) Data Handling within the International VLBI Service. Data Sci J 12:WDS81WDS84. doi:10.2481/dsj.WDS-011

Bizouard C, Gambis D (2011) The combined solution C04 for Earth Orientation Parameters consistent with International Terrestrial Reference Frame 2008. https://hpiers.obspm.fr/iers/eop/eopc04/ C04.guide

Bradley BK, Sibois A, Axelrad P (2015) Influence of ITRS/GCRS implementation for astrodynamics: coordinate transformations. Adv Space Res. doi:10.1016/j.asr.2015.11.006

Brzezinski A (2012) On estimation of high frequency geophysical signals in Earthrotation by complex demodulation. J Geodyn 62:74-82

Haas R, Sekido M, Hobiger T, Kondo T, Kurihara S, Tanimoto D, Kokado K, Wagner J, Ritakari J, Mujunen A (2010) Ultrarapid DUT1-observations with E-VLBI. Artificial Satellites 45(2/2010):75-79
Hobiger T, Otsubo T, Sekido M, Gotoh T, Kubooka T, Takiguchi H (2010) Fully automated VLBI analysis with c5++ for ultra-rapid determination of UT1. Earth Planets Space 62:933-937

Kareinen N, Hobiger T, Haas R (2015) Automated analysis of Kokee-Wettzell Intensive VLBI sessions-algorithms, results, and recommendations. Earth Planets Space 67:181. doi:10.1186/ s40623-015-0340-x

Kareinen N, Hobiger T, Haas R (2016) Automated ambiguity estimation for VLBI Intensive sessions using L1-norm. J Geodyn. doi:10. 1016/j.jog.2016.07.003

Kokado K, Kurihara S, Kawabata R, Nozawa K (2012) Recent activities of Tsukuba Correlator/Analysis Center. In: Behrend D, Baver KD (eds) Proc. 7th IVS General Meeting. NASA Conference Publication, NASA/CP-2012-217504, pp 105-114

Koyama Y, Noriyuki Kurihara N, Tetsuro Kondo T, Sekido M, Takahashi Y, Kiuchi H, Heki K (1998) Automated geodetic very long baseline interferometry observation and data analysis system. Earth Planets Space 50:709-722

Koyama Y, Kondo T, Sekido M, Hobiger T, Takiguchi H, Wada K, Kurihara S, Haas R, Wagner J, Mujunen A, Ritakari J (2010) Ultra rapid dUT1 estimations from E-VLBI Sessions. Advances in Geosciences, vol. 20 (Solid Earth). World Scientific Publishing Company, pp 197-204

Luzum B, Nothnagel A (2010) Improved UT1 predictions through low-latency VLBI observations. J Geod. doi:10.1007/ s00190-010-0372-8

Matsuzaka S, Shigematsu H, Kurihara S, Machida M, Kokado K, Tanimoto D (2008) Ultra rapid UT1 experiment with e-VLBI, In: Finkelstein A., Behrend D (eds) Proc. 5th IVS General Meeting. Saint Petersburg, pp 68-71

Matsuzaka S, Kurihara S, Sekido M, Hobiger T, Haas R, Ritakari J, Wagner J (2010) Ultra-raid dUT1 Measurements on JapanFennoscandian Baselines-Application to 24-hour Sessions. In: Behrend D, Baver KD (eds) Proc. 6th IVS General Meeting. NASA Conference Publication, NASA/CP-2010215864, pp 143-147

Nothnagel A, Behrend D, Bertarini A, Charlot P, Combrinck L, Gipson J, Himwich E, Haas R, Ipatov A, Kawabata R, Lovell J, Ma C, Niell A, Petrachenko B, Schüler T, Wang GL (2016) Strategic Plan of the IVS for the Period 2016-2025. In: Proc. 9th IVS General Meeting, in press. http://ivscc.gsfc.nasa.gov/about/strategic/ index.html

Petrachenko B, Niell A, Behrend D, Corey B, Böhm J, Charlot P, Collioud A, Gipson J, Haas R, Hobiger T, Koyama Y, MacMillan D, Malkin Z, Nilsson T, Pany A, Tuccari G, Whitney A, Wresnik J (2009) Design aspects of the VLBI2010 system. NASA/TM-2009-214180, Washington, DC, USA, p 58

Petrachenko B, Bertarini A, Alef W, Behrend D, Cappallo R, Hase H, Ma C, Niell A, Nothnagel A, Zhang X (2014) VGOS Data Transmission and Correlation Plan (Version: November 18, 2014). Internal Report, VGOS Project Executive Group (VPEG). http:// ivscc.gsfc.nasa.gov/technology/vgos-documents.html

Sekido M, Takiguchi H, Koyama Y, Kondo T, Haas R, Wagner J, Ritakari J, Kurihara S, Kokado K (2008) Ultra-rapid UT1 measurements by e-VLBI. Earth Planets Space 60:865-870

Tsunami UDP protocol (2009) http://tsunami-udp.sourceforge.net/ 